\title{
Producción bibliográfica en ciencias sociales, como insumo para la construcción de categorias sintéticas para la comprensión del desarrollo de la sociologia paraguaya
}

\author{
The bibliography production in social sciences and input for the construction of \\ synthetic categories for the understanding of the development of the Paraguayan \\ sociology
}

\section{Javier Numan Caballero Merlo ${ }^{1}$}

Resumen: La producción de conocimiento en cada comunidad nacional tiene sus particularidades de gestación y desarrollo según su propia historia y sus circunstancias. La del desarrollo de la sociología paraguaya no es por tanto, la excepción. Pero asimismo, se reconoce que ciertas estructuras, instituciones y condiciones socio históricas macro producen desarrollos particulares nacionales dentro de dichas circunstancias comunes. Por lo cual, los procesos de ciertos desarrollos tienden puentes de conexión, posibilitan el entramado, comunes denominadores aunque en cada pais se le imprima, claro está, su realidad sui generis. Los estudios sobre la vinculación entre el 'tipo' de producción bibliográfica y el desarrollo de las ciencias sociales son asi compartidos casi sin excepción por los investigadores latinoamericanos. Distinción entre un tipo de producción de cariz más anecdótico, ensayístico, por un lado, y otro más sistemático. Uno y otros, ligados sin embargo, aún en tiempos históricos superpuestos y hasta compartidos, con la creación de universidades y de centros privados de investigación, y con la ausencia de dichos espacios institucionalizados. Se describe la producción bibliográfica registrada exhaustivamente entre un articulo seminal al respecto (Heisecke, 1965), hasta un segundo (Meliá y Palau, 1975) que incluye comentarios sobre el primero. Hago en esta segunda entrega una revisión de éste último, agregándole de manera original su contextualización y análisis sintético interpretativo desde categorías de análisis sociológico sistematizadas de la teoría social latinoamericana, aplicada a la comprensión de los desarrollos de las disciplina según el tipo de producción, determinada asimismo según el tipo de vinculación con desarrollos institucionales diferenciales, por su vez dependientes del desarrollo histórico de la sociedad en cuestión.

Palabras clave: Producción, bibliografía, desarrollo, sociología.

Abstract: The production of knowledge in each national community has its peculiarities of conception and development depending on its own history and its circumstances. The

\footnotetext{
${ }_{1}$ Máster en Sociologia. Candidato a Doctor por el Programa de Doctorado en Ciencias Sociales de la UNAM, Posadas - Argentina. Investigador Categorizado PRONII - CONACYT y Docente Investigador por la Universidad Autónoma de Asunción (UAA), Departamento de Postgrado e Investigación. E-mail: javiernuman18@hotmail.com

Recibido: 17/07/2014; Aceptado: 10/11/2014.

http: / / dx.doi.org/ 10.18004/riics.2015.julio.115-129
} 
development of the Paraguayan sociology is therefore not the exception. But it is also recognized that certain structures, institutions and socio historical conditions macro produce particular national developments within these common circumstances. Therefore, the processes of certain developments tend connection bridges, enable the network, common denominators although in each country it is stamped, of course, its sui generis reality. The studies about the link between the 'type' of bibliographical production and the development of the social sciences are thus almost without exception shared by Latin American researchers. Distinction between a more anecdotal, essay type of production, on one side, and another one more systematic. One and others, related however, even in overlapping historical times and even shared, with the establishment of universities and private research centers, and with the lack of those institutionalized spaces. One and others, connected, however, even in overlapping historical times and even shared, with the establishment of universities and private research centers, and with the lack of such institutionalized spaces. It is described the bibliographical production recorded extensively from an article to the seminal (Heisecke, 1965) to a second one (Meliá and Palau, 1975) containing comments on the first described. I make in this second installment a review of the latter, adding in an original way its contextualization and synthetic interpretative analysis from systematized categories of sociological analysis of the Latin American social theory applied to the understanding of the development of the discipline according to the type of production, also determined depending on the type of linkage with differential institutional developments, in turn dependents of the historical development of the society at issue.

Keywords: Production, bibliography, development, sociology.

\section{INTRODUCCIÓN}

Como investigador ${ }^{2}$, aparte de las exigencias institucionales de probar rendimiento, producción objetivada y visibilidad, se tiene el desafio personal y académico de inscribirse o desarrollar una línea de investigación ${ }^{3}$ que sea a la vez fructífera y necesaria. He intentado por largos daños, en circunstancias contextuales e institucionales adversas conciliar ambas exigencias, las externas y las propias. La realidad del país ha ido cambiando, lenta y casi inexorablemente, y hoy, el contexto permite en condiciones diferentes, entre otras, con la creación del Programa Nacional de Incentivo a los Investigadores (PRONII) contar con el incentivo que permite aun con ciertas limitaciones, constituir un espacio temático como línea de investigación. Y desarrollarlo con estudios sistemáticos, lectura, trabajo empírico sostenido y abierto, y cierta garantía de hacerlo público y visible a

2 Categorizado dentro y por el Programa PRONII del CONACYT, periodos continuos del 2011-2013, y 20142016. Y, Docente Investigador por el Departamento de Postgrado e Investigación de la Universidad Autónoma de Asunción (UAA).

${ }^{3}$ Línea de Investigación en Docencia e Investigación en Historia Sociológica de la Sociología en Paraguay. 
través de las pocas publicaciones regulares con formato de revista, existentes dentro de fronteras. Es una forma de compartir (lo), hacer visible todavía un trabajo arduo y silencioso, muy solitario, y por tanto siempre parcial. Las dificultades en la constitución de equipos de trabajo colectivo, y división del trabajo retardan la utopía, que ya contempla en el proyecto, un programa de investigación al estilo lakatosiano ${ }^{4}$. El paso de la tarea del archivista se hace así necesario y fundamental, recopilando, registrando y sistematizando todo el material primario y secundario que se pueda referente a nuestro tema problema inaugurado ya con ciertos trabajos, el del desarrollo de las ciencias sociales en Paraguay, particularmente el de la Sociología. Este artículo, constituye una segunda entrega en esta misma revista y dentro de la misma línea de investigación a sustentar.

El contexto general es el de la modernización capitalista, que en la experiencia latinoamericana se impone con ritmos diversos entre Centroamérica y Sudamérica, y dentro de esta, a Paraguay, de los demás miembros del Mercosur. Modernización demorada y conservadora, con la herencia de una larga dictadura, han jugado en contra de un desarrollo fuerte de una vida universitaria conectada con los problemas que hacen justamente a la oferta de la Sociologia como disciplina sistemática. Debilidad institucional acorde con un contexto autoritario, de tránsito lento y conservador de un modelo rural oligárquico a una sociedad modernizada en vías de desarrollo, en definitiva, tanto sobre quienes pueden interesarles llevar adelante la tarea -como oferta; resolución de problemas; hacedores de políticas, etc.- como sobre qué y para qué realizarla -problemas sociales arquetípicos que demandan el desarrollo de una sociología.

Al respecto, en una entrevista con un destacado colega sociólogo5, éste rescata que el prestigioso profesor argentino Francisco Delich, después de su estadía en Paraguay por finales de los años '70, y particularmente como Director de un curso de postgrado en sociología rural dentro del Centro Paraguayo de Estudios Sociológicos (CPES) asociado con un centro regional latinoamericano- publica un artículo titulado "El despotismo agrario republicano" en la revista 'Estudios Rurales Latinoamericanos'. Será ahí, nos recuerda Céspedes, que por primera vez se aplicará a Paraguay, a través de Delich, una expresión de Kalman Silvert6 que dice, que 'Paraguay es un cementerio de teorías'. Célebre frase entre pocas provenientes de las ciencias sociales, esgrimida en diversas tertulias pseudo intelectuales y politicas locales.

${ }^{4}$ Concepto de Imre Lakatos, en su metodología de los programas de investigación.

5 De Maria Lilia Robledo Verna al Ms. Roberto Céspedes. Asunción, sábado 13 de junio de 2009, 10.30 hs.

6 Un académico norteamericano, latinoamericanista de la década del 60, comienzo de los 70 . 
Detalle, frase aislada, y fuera de contexto, desconsidera aquello para la cual fue producida por Silvert y asimismo citada por Delich. La referencia de estos dos destacados profesionales de las ciencias sociales hace referencia a que equivocadamente se insiste en incluir a Paraguay como sí formando parte de una historia común de desarrollo del Cono Sur -matriz histórica, socio cultural, política y económica-, y por tanto, hasta ahora se continúa intentando aplicar las mismas teorias que se aplican a los demás países del Cono Sur como ser Uruguay, Argentina y Chile para el caso paraguayo. Pero tal aplicación obvia que la modernización en uno $u$ otro caso dista de ser homogénea y es por lo demás sumamente diferente, haciendo espuria tanto la generalización de la aplicación de teorias, como la conclusión de cementerio de teorias. La premisa histórica falsa de partida invalida en la experiencia tal conclusión. Doble error, en la presunción punto de partida, y en la aplicación de teoría fuera de contexto.

La realidad social sudamericana fuera Paraguay es fundamentalmente urbana (y portuarias marítimas), producto de una temprana modernización ya desde finales del siglo XIX. Así como con la experiencia histórica de haber perdido sus democracias. Mientras, en Paraguay, la sociedad dislocada ${ }^{7}$ y dual el ámbito rural tradicional continúa teniendo un gran peso socio cultural, económico, y político. Y su realidad política aparece marcada por la experiencia histórica autoritaria de larga data, con fuerte presencia y peso del estamento militar, y un aislamiento secular que recién irá moldeando su experiencia democrática en condiciones más favorables a partir de 1989 con el fin de la dictadura stronista.

Hasta ahora, serias dificultades vinculadas con el modelo económico y político del proyecto país dificultan el desarrollo de una burguesía emprendedora. Lejos de ser cementerio de teorias, la aplicación fuera de contexto o mal uso casi ya como un refrán muletilla anecdotario jocoso, o tragicómico, de querer justificar que Paraguay está más allá del bien y del mal, donde nada funciona, se entiende o se puede aplicar. Para que todo siga igual. La afirmación no solo es falsa sino que politicamente desmovilizadora y conservadora. Como sea, por desconocimiento o perversión, nos lleva a estar atentos evadiendo la trampa definida además a priori de segura tumba para cualquier comprensión crítica, y que para entender a Paraguay en cualquiera de sus dimensiones, hay que partir de una dialéctica concepto experiencia considerando su realidad empírica e histórica, sin imponerle modelos teóricos mecánicamente sin ninguna conexión con su experiencia.

En este trabajo podemos encontrar coincidencias con las propuestas de diagnosis de varios autores latinoamericanos respecto a la distinción entre los

7 Hacemos referencia a la obra del mismo nombre de Luis Galeano. 
abordajes de los temas sociales desde la perspectiva del ensayo o de una sociología más sistemática (Schuttenberg, 2006; Reyna, 2004; Pereyra, 2007, 2008, 2010; Neiburg y Plotkin, 2004; Noé Miño y Dávila, 2005; Murga Frassinetti y Boils, 1979; González, 2000; Trindade et al., 2007; Blanco, 2004; Bruschera et al., 1969; Buccafusca et al., 2000; Caballero Merlo, 2011; Devés Valdés, 2001, 2003, 2004, 2007).

Transformándose el ensayo y lo sistemático en dos categorias que permiten a la vez sintetizar y ordenar la dispersión de estudios sobre los más variados tópicos y desde disciplinas sociales diversas, así como hacer comprensibles su orientación $\mathrm{y}$ por tanto el alcance en uno u otro caso.

En nuestra primera entrega al respecto del desarrollo de la Sociología, relacionando producción de conocimiento bibliográfica con etapas, sintetizamos de manera critica el valioso aporte de Heisecke (1965). Ahora, nuevamente, desde la producción sociológica y su historia, presentamos un segundo gran esfuerzo inicial, el de Meliá y Palau (1975). Que con el primero cronológicamente comparten el mérito de 'saturar' el registro de obras y temas hasta entonces, poniendo asimismo en discusión su interpretación. Ambos pusieron en marcha esa recuperación de memoria hacia el interior de la propia producción nacional de tinte sociológico, de una forma sistemática, tarea a que nos sumamos. El aporte de ambos estudios es de utilidad innegable, posibilitando acumular una base de datos sobre la cual trabajar, yendo más allá del límite de la descripción, con la ventaja del pasar del tiempo, de manera más teórica y sintética.

\section{Relevamiento bibliográfico y su conexión con el cariz de la producción sociológica}

Analizando comparativamente y dentro del contexto latinoamericano, los autores, destacan que el volumen de la producción sociológica en Paraguay hasta la fecha en cuestión (1975), así como la continuidad en el tratamiento de sus problemas no expresa un acervo significante. Esto se contradice aparentemente prima facie con la contextualización histórica realizada tanto contemporáneamente y en el artículo mencionado anteriormente de Heisecke desde el CPES (1965) -cuya obra se cita y referencia en la página siguiente; así como con la más lejana de Justo Pastor Benítez (1996/1955 - Formación social del pueblo paraguayo $\left.{ }^{8}\right)$, asî como por la propia constitución del CPES (1964) como centro y dínamo al menos,

\footnotetext{
${ }^{8}$ Ver asimismo, del autor: Sociología paraguaya. Rio de Janeiro, 1961. Ob. Cit., Meliá y Palau, 1975 , p. 174.
} 
como necesaria referencia en su cabalgata solitarita en un contexto dictatorial, y sin dejar de lado asimismo la creación de la UCA (1960) y su Carrera de Sociología (1972).

Según los autores, sin trabajos con aportes desde el materialismo dialéctico, sus desarrollos se han producido dentro de un espacio teórico muy restricto entre un "incipiente" desarrollismo, y una dominancia del liberalismo. O sea, existe algún vínculo con la teoria social general y con la latinoamericana, esta línea es la queremos explorar, utilizar para construir sintesis.

Entre las razones que imposibilitaron generar una producción sostenida y significativa en sociología se mencionan: la ausencia de centros de estudio y formación el disciplina; la escases de recursos humanos capacitados; oportunismo individual; y la total indiferencia de las instituciones tanto públicas como privadas hacia la consideración e inversión de las ciencias sociales. Situación que aún hoy creemos no ha cambiado de manera sustancial, ya que se investiga mayormente como producto asociado a consultorias, donde hay recursos, se definen las lineas de pesquisa, así como sus temas-problemas-objeto de estudio y sus marcos teóricos. Es un "oportunismo" que va acumulando un perfil de la nueva producción sociológica en el país, ligada a organismos gubernamentales, internacionales y ONGs. Es el camino de los recursos, no necesariamente coincidentes con el de una sociología sistemática, académica (diferente de academicista), lo que va dibujando el camino aparentemente caprichoso de las temáticas abordadas y las publicaciones que se hacen visibles.

De esta forma, la producción de conocimientos en sociología separada de ligazones institucionales con una clara autonomia de gestión y de definición, va diseñando una muestra a veces cambiante, totalmente parcialista, puntual, empiricista, de corto alcance, y muy anárquica: “(...) el panorama de la sociología paraguaya se presenta como una suma de elementos dispersos, con extrañas ramificaciones, (...)". (Meliá y Palau, 1975, p. 147) Lo cual, contingente e históricamente -aquello del hombre y sus circunstancias-, va generando cierto perfil a los desarrollos de la Sociología en Paraguay, con aportes desde otras disciplinas no específicamente sociológicas, como ser la historia social, el ensayo y la antropología social. Esto aproxima el abordaje de Meliá y Palau de entonces con el nuestro y los autores latinoamericanos referenciados más arriba, considerando los temas, las disciplinas y en palabras de los dos autores, la convivencia entre un enfoque más sociográfico y otro desde la tradición nacional. El primero podriamos asociarlo hipotéticamente con el sistemático y el segundo con el de corte más ensayístico, cosa que hacemos como propuesta además, que permite trazar 
paralelismos de manera comparativa con los desarrollos de la sociología en los demás países latinoamericanos.

Podriamos, y debo agregar, tampoco de los lugares institucionales privilegiados de producción de conocimiento típicamente sociológicos, como lo ha sido por toda América Latina, el espacio social de la Universidad. Hasta ahora, la misma está prácticamente ausente en la investigación y publicación de manera tanto significativa como sistemática. Una tradición más áulica, que aún le cuesta definir y asumir líneas de enseñanza e investigación, así como de construcción de una política de extensión ligadas con esas instancias, desde precisos lineamientos epistémicos, teóricos, metodológicos, y técnicos. A lo cual habría que agregar la insoslayable inserción de la Universidad en la problemática nacional, es decir, de la relación dialéctica entre universidad y sociedad. Lo cual, debería igualmente poder definir los temas problemas más tratados, investigados, así como los desarrollos teóricos metodológicos y técnicos más apropiados y desarrollados al efecto. Formación proceso de enseñanza aprendizaje, malla curricular, práctica universitaria, extensión, temas de tesis, producción de tesis, artículos, congresos, orientadores especializados, etc., pero no como simple juego del mercado, o de la tensión entre oferta y demanda, sino como conocimiento producto de la realidad social, sus procesos, e historia. Las ONGs y otro tipo de instituciones privadas, o de estatus mixto, se han desarrollado fuera de la Universidad y realizan gran parte de esta labor, de manera mucho más empiricista efectista, con agendas y financiamiento que condicionan igualmente el carácter de sus producciones e intervención sociales.

\section{Producción sociológica en los últimos años}

Los autores se enfocan en dos críticas al trabajo ya referenciado de Heisecke. Una más velada, que se explícita más adelante en el mismo (a), y otra más concreta, que lamentablemente cae en lo mismo que señala, enuncia pero no profundiza (b):

a) Ponerle fechas, trabajos (obras específicas) y autores a diversas etapas en el desarrollo de la sociología paraguaya; y,

b)Mayor pecado aún, parece cometer Heisecke, al considerar que la última etapa desarrollada contemporáneamente a la redacción de su artículo, se asocia y describe como de tránsito hacia la sociología científica, particularmente con la creación del CEPES. Con el agregado, que es en parte objetivo, que lo hace sin entrar a analizar qué componentes hacen a dicha 
producción ahora científica así como de la a-cientificidad de la "tradición" sociológica paraguaya desarrollada hasta ese entonces.

Aprovecho la oportunidad para agregar otras críticas, válidas para ambos estudios, que me parecen necesarias operativamente al contar con la ventaja de disponer de más material para abstraer variaciones, dado el objetivo de ir más allá del registro, del archivo, de lo arqueológico ${ }^{9}$ descriptivo, buscando conexiones y sentido. La ausencia de teoria y categorias que hubieran evitado caer en la trampa de perderse en la descripción de la dispersión empírica, a modo de coleccionistas de datos, que le diera sustento de organización de los mismos con ciertos criterios que permitieran asimismo su comprensión sin forzar la imposición de etapas de manera más bien ideal-formal.

Frente a la dispersión y ramificación aparente sin ton ni son, para eso está la teoría y ciertos criterios construibles desde las mismas que permiten abstraer alguna generalización y posibilidad de síntesis. Es cierto, como ya dicho más arriba, que ya en el trabajo de Meliá y Palau, con más ventaja temporal de acumulación de producción y, por tanto, de perspectiva que Heisecke, intentan explícitamente trazar paralelismos analíticos precedentes en la producción latinoamericana, como ser la identificación teórica ideológica de las publicaciones para situarlas dentro de una u otra perspectiva típica; o bien, cosa que utilizan en su estudio como herramienta y criterio simultáneamente, el de incluir los trabajos dentro de determinadas temáticas 'atravesadas' por diversas disciplinas, como más abajo se detalla "se ha preferido ordenar estas notas sobre la base de un orden más interno cual es el de las líneas principales en que de hecho se han producido publicaciones" (Meliá y Palau, 1975, p. 149). Intento de organización, después del registro, desde los temas (migraciones, educación), sus líneas principales, las disciplinas como bloques organizativos (historia social, ensayo), tendencias desde la sociografia y tradición nacional, la no identificación clara con 'escuelas' que definen ideológicamente la producción sociológica, ni etapas, ni épocas.

O sea, intentaron varias vias, que hoy podemos lograr sintetizar de manera más abstracta con el auxilio de la teoría dicha organización todavía muy laxa y hasta imprecisa. Nunca existen épocas u etapas a priori, los investigadores las construyen tomando ciertos recortes de la experiencia desde algún cuerpo conceptual, cuando dicha experiencia lo permite, no es cuestión de mera imposición, y hoy creo que, se pueden presentar etapas con ciertos énfasis, sin pretender pasar el tipo por la experiencia siempre menos definido. Por ello, Meliá y

${ }^{9}$ Siguiendo la propuesta y búsqueda ulterior de Michel Foucault. 
Palau no solucionan el problema teórico, pero les permite encontrar un criterio de organización del material.

El sintetizar los temas es importante pues tenemos un panorama descriptivo de lo que se trata en Paraguay, pero también es relevante explicar por qué, y el tipo de conocimiento producido (ensayo-sistemático), así como sus enfoques teóricos y que puedan tipificarse como sociológicos. Si todo vale, y todo entra bajo la égida de la sociología entonces su particularismo interpretativo y explicativo pierde significación analítica.

Por otro lado, con todo lo de apologético y unilateral que pueda resultar, así como percibir el sesgo y parcialidad en su descripción e interpretación, ambas muy selectivas marcando diferencia, siempre, dramáticamente a favor de ensalzar al CPES, son comprensibles en Heisecke dada su vinculación con el CPES, la búsqueda de su destaque, que lo llevan a asociar casi el nacimiento de la disciplina para el país, con el CPES y la figura del Dr. Domingo Rivarola casi a imagen y semejanza del Instituto de Sociología Gino Germani en la UBA.

\section{Tendencias formales de la sociologia paraguaya}

Partiendo de un "es indudable" (no muy sistemático) que la producción sociológica presenta dos tendencias formales bien definidas: una sociográfica, de carácter descriptiva y funcionalista; y, dentro de lo que se considera la prolongación (no se dice desde dónde o cuando, o con qué autores y obras) de una tradición nacional (entonces habría una) en escritos de historia social, ensayos socio culturales, y la investigación antropológica. Introduciendo 'dos variables explicativas fundamentales' según los autores, la de proceso histórico y la de totalidad -como todo complejo conflictivo y hasta contradictorio pero dialécticamente integrado. Me animo a afirmar aquí, que se dicotomizan las posiciones entre funcionalismo y naturalismo dialéctico (materialismo histórico), pero creo que por la producción bibliográfica, si bien lo primero podriamos conceder hasta como primado hegemónico, hasta hoy en día, de lo segundo existen muy pocas muestras y/o referencias al respecto, tal vez por la lente de los autores del artículo, y asimismo más animados por prácticas discursivas que con obras producidas desde un marco conceptual marxista.

Contradiciendo además, en cierta forma, lo que se dice en los primeros párrafos de la página 147 (Meliá y Palau, 1975), cuando se señala que: "No se encontrarán, por supuesto, análisis que utilicen las categorias del materialismo dialéctico ni que estudien los procesos sociales en términos del concepto marxista 
de lucha de clases sino rara o tangencialmente". Lo que objetivamente valida a nuestro análisis crítico, y muestra ciertas contradicciones respecto a los abordajes de las producciones cuanto a la definición o no de etapas y tradiciones en el desarrollo de la sociología paraguaya.

Nuestra propuesta, encima del análisis, y respuesta, es que son identificables las orientaciones de las producciones y junto con las mismas con otros criterios, etapas o discontinuidades objetivables.

\section{Limites y alcances de la producción sociológica anotada}

Se discuten y definen ciertos criterios de organización del material existente, necesarios dada la fragmentación ya apuntada de la producción. Por ello se afirma que parecen irrelevantes tanto los paradigmas más usados tanto temporales como temáticos.

Asimismo, se afirma que no se dan "épocas ni etapas" claramente diferenciadas en la producción sociológica nacional, utilizando como criterio de distinción, que formas y métodos tradicionales continúen vigentes en el presente sin verificarse una efectiva sustitución por otros nuevos desarrollos.

Creemos, afirmativamente, que siempre se pueden establecer y visualizar alguna serie de características o criterios que de manera articulada permitan trazar cierto perfil aunque general, de algo así como etapas u épocas de la producción, prácticas, desde y dentro de alguna disciplina como el caso de la sociología. Sino, de hecho, estaríamos negando si se quiere el propio particularismo que define a la sociologia, es decir, el influjo de la sociedad en sus productos y la forma de su producción. Una época, en un sentido amplio que contemple procesos y espacios nacionales, regionales e internacionales, así como las especificidades de las formas de relacionamiento y los productos resultantes de las mismas en distintos ámbitos -cultural, política, economía- asimismo, constituyen los elementos necesarios y suficientes para describir inscribiendo dentro de una totalidad e historia, y pudiendo explicar la significación contextualidad (dentro y en relación dialéctica con un orden social) de las producciones sociológicas, obras, autores, orientaciones, fuentes, etc.

Además, la superposición de formas y métodos tradicionales (sean éstos cuales fueren en su momento y dentro de un cuadro más amplio que los ubique) siempre han convivido con aportes más antiguos y otros más nuevos, algunos hasta revolucionarios, sin que ello constituya un impedimento para distinguir épocas o etapas, por el contrario, las clarifica, aunque claro está, se deba 
contemplar, que en un misma etapa, donde domina una forma o método, temas u marcos teóricos determinados, también se desarrollan de forma simultánea otras opciones.

Nunca ha habido ninguna continuidad o discontinuidad histórica con un monólogo o exclusivismo objetivo, a no ser, con la imposición de vacíos, silencios, o sea, de imponer exclusiones, que de por sí, asimismo, darian material para definir la orientación de una etapa, o hegemonía de determinadas perspectivas positivismo, funcionalismo, a-historicidad, autoritarismo, etc.

\section{Distribución temática de la producción sociológica paraguaya}

Se destacan las dificultades justamente de construir criterios de acumulación, sistematización y diferenciación de la producción en nuestra área de estudio: “(...) resultaría sin sentido por incoherente y atomizante, querer cubrir todas las secciones en que se suele repartir una sociología; (...)." (Meliá y Palau, 1975, p. 148) Dimensiones de la sociologia paraguaya que no cuentan con un cuerpo de publicaciones suficientemente amplio para que se justifique su tratamiento sistemático; mientras otras problematizaciones si lo ameritan, la idea de los autores es concentrarse en las mismas, y no en todo lo que exista con la consiguiente dispersión de materiales, de posturas, y de forzar articulaciones entre las mismas, que fuercen abrir demasiado el abanico del análisis. Esto es elucidativo por sí mismo, se constituye en un dato, pues es el estado de situación con el que se deparan hasta el año de 1975.

También un problema ya destacado más arriba, asumen no poder dar un tratamiento sistemático, yo creo que hoy si se puede, reconociendo todas las dificultades y debilidades anotadas, ese es el desafio a la invocación de la teoría e imaginación sociológica. Siempre se toman decisiones desde algún lugar aunque sean implícitos, la labor es sistematizarlos explícitamente.

\section{Los tiempos y por qué de los mismos}

Partiendo de un cuasi reconocimiento de la existencia de trabajos anteriores que han caracterizado las lineas y tendencias de la sociología paraguaya e incluyendo un relevamiento bibliográfico básico se proponen tratar la producción bibliográfica de carácter sociológico desde los años 60 hasta su presente (1975). Ligados a los años '60 con la fundamentación de la creación de diversas instituciones que en grados diversos contribuyeron al influjo de la producción y 
publicación en dichas áreas. Década asociada asimismo con la incorporación de métodos y técnicas de carácter más sistemático empírico sociales. Esto no dista en casi nada de la perspectiva de Heisecke, solo que se hacen desde diferentes lugares, aunque viven los mismos años, y hasta pasaron por las mismas experiencias institucionales (Palau -ahora Base Is, antes CPES al igual que Heisecke).

\section{Lo sociológico fuera de la sociologia}

Se trabaja encima de todas aquellas producciones definidas como significativas dentro del período delimitado, pero que provienen de ámbitos que por sus sustentos teóricos y metodología -histórico y filosófico- no siempre se considerarian sistemáticos (científicos) de parte de la sociología académica, como lo son los aportes de: historia social, ensayo socio-cultural, estudios de antropología y sociolingüistica. El análisis social en Paraguay, parece asociarse por los comentaristas, como privilegiado a través de esos caminos. Problema que subsiste hasta nuestros días, qué es sociológico y qué no lo es, mismo desde los centros privados que dicen dedicarse a ello, o profesionales que se definen como practicantes de la sociología.

Se destaca que la mayoría de los aportes sociológicos en la primera mitad del siglo veinte provienen de un 'estilo más literario', ensayístico - de influencia didáctica ejercida hasta en el presente, en el decir de Meliá y Palau (1975, p. 155). Que 'de informe técnico', y con un fuerte componente que apunta a la conciencia nacional. Pero, entre los diversos autores de referencia que se analizan aquí, existen variaciones y contradicciones en cómo tomar o definir a algunos de los más notorios autores del período, algunos de corte más ensayístico (Meliá y Palau, 1975), para otros con claros signos de orientación positivista (Heisecke, 1965; Caballero, 2011), y hasta la con-fusión entre unos y otros (autores y obras) y solapamiento de etapas.

Por su parte, desde la antropología social, se ilustra respecto a qué temas eran los más trabajados por y a hasta entonces. Reiteración temática, que nos direcciona en nuestra pesquisa, viendo que, la cuestión rural, y la cuestión indigena, independientemente de su respuesta en políticas, son dos de los grandes ejes temáticos recurrentes abordados por las ciencias sociales en Paraguay. El tema de las Reducciones Jesuíticas por un lado, y el del nacionalismo por otro, son otros de los temas recurrentes. 


\section{La sociologia descriptiva}

Interesante es la asociación que podemos deducir hacen los autores, entre una sociología sistemática, de carácter cuantitativo y técnico, asociada a la llegada de investigadores extranjeros, principalmente norteamericanos, cuya recolección de datos apuntaba a dos objetivos principales: la realización de sus trabajos académicos en sus países de origen; o, en el carácter de consultores, para la elaboración de Informes "Técnicos" para organismos internacionales, gubernamentales o consultoras privadas. Sociología sistemática y técnica, que además se presenta como embazada en el marco teórico funcionalista, y como si fuera poco, influyendo en los investigadores locales que se incorporan, principalmente a: organismos gubernamentales como la Secretaria Técnica de Planificación (STP), instituciones privadas como el CPES o el Instituto de Desarrollo Integral Armónico. Sociología 'técnica' supuestamente neutral, asociada a la modalidad del Informe, como síntesis descriptiva (Meliá y Palau, 1975 pp. 162163). Esto constituye un dato muy relevante para la investigación de más largo alcance que llevo a cabo, más, para el carácter fundacional del CPES.

Respecto a los estudios de carácter demográfico, una referencia necesaria sobre los estudios de población, definiendo para algunos un antes y un después de la sociología paraguaya, está la obra titulada "Población, urbanización y recursos humanos en el Paraguay", editado por Rivarola y Heisecke (CPES, 1969/1970). Sus ligazones con lo social, económico e histórico, lo hicieron en su momento, un material de referencia obligada y muestra a su vez de la nueva sociología paraguaya.

Por otro lado, es importe el tópico que registra los estudios sobre estratificación, movilización y cambio social. Pues se comienza por asociar algo de nuestro interés particular en el presente estudio, ya que, coincide el lanzamiento del $1^{\circ}$ número de la Revista Paraguaya de Sociología, no por acaso, creemos, con la publicación en la misma de un artículo en 1964 de su Director Domingo M. Rivarola sobre la movilidad social horizontal en el Paraguay. El trabajo aborda las migraciones internas y su relación con el cambio social, introduciendo en el Paraguay, según los comentaristas, "un tema que ha dominado ampliamente la sociología latinoamericana de la década del 60." (Meliá y Palau, 1975, p. 169). Abriéndose así una serie de trabajos del mismo y otros autores nacionales sobre el tema. Se insiste en que la regla son abordajes instrumentales, planificadores racionalizadores- del desarrollo, programación del desarrollo regional, dentro de los modelos teóricos desarrollistas que ni siquiera llegan al punto de la reforma. 


\section{Conclusiones parciales de un programa de investigación abierto}

Lo que nos interesa no es la dispersión de los datos, ni tanto la descripción de los mismos en sí misma, a pesar de su necesaria e innegable utilidad para el diagnóstico, cuadro de situación y material empírico al fin, con el cual hacer posible y llevar adelante el esfuerzo fértil o no, de la sintesis teórica. Datos, necesarios para poder visualizar lo oculto, por ejemplo, que hasta la década del '70 del siglo XX, no se puede negar aunque se quiera por diversas intereses -de competencia interinstitucional, politicas de gobierno de turno, o cuestiones personales-, que el CPES se constituyó en el eslabón de acopio y sistematización de estudios, informaciones y referencias respecto a la producción en ciencias sociales en general y en sociología en particular, en el ámbito regional y nacional. Lo más destacable así, es que hasta dicha década, desde 1964, el archivo necesario ha sido el CPES. Se destaca que hasta los '60 y '70 realmente es muy poco lo que se puede encontrar con un perfil típicamente sociológico, o su revés, sintomatizando mucho de lo que no lo era.

Este trabajo nos da una idea del volumen-cantidad, áreas-temáticas de énfasis, instituciones o medios de publicación, y los períodos en términos cronológicos, todo lo cual es fundamental como criterios para nuestros objetivos de recomposición de etapas, ciclos y perfil de sus orientaciones del pensamiento social/sociológico en Paraguay.

Quedamos en deuda con ese registro total, del cual el presente artículo constituye un pequeño aporte, pero abriendo por programa y campo de investigación estable, el de continuar con la sistematización, cronológica, institucional, y bajo otros criterios de la producción en ciencias sociales, en particular de Sociología, línea de investigación que pensamos continuar explorando y explotando en el futuro.

\section{REFERENCIAS}

Blanco, A. (2004). La sociología, una profesión en disputa. En Neiburg, F. y Plotkin, M. (Ed.), Intelectuales y expertos: La construcción del conocimiento social en la Argentina. Buenos Aires: Paidós.

Bruschera, D., Germani, L., Maggiolo, P., y Ribeiro, T. (1969). Situación y destino de América Latina. Montevideo: Fundación de Cultura Universitaria.

Buccafusca, S., Serulnicoff, M. L., y Solari, F. (2000). Temperaturas de época: Gino Germani y la fundación de la carrera de Sociología. En González, Horacio. (Compilador), Historia crítica de la sociología argentina: Los raros, los clásicos, los científicos, los discrepantes. Buenos Aires: Colihue Universidad Sociología. 
Caballero Merlo, J. N. (2011). Cien años de desarrollo de la sociología en Paraguay en el año de su Bicentenario: Del rezago histórico institucional en el pasado a la debilidad contemporánea. Revista Internacional de Investigación en Ciencias Sociales, 7(2), 119-160.

Devés Valdés, E. (2001). El pensamiento latinoamericano en el siglo XX: Del Ariel de Rodo a la CEPAL (1900-1950) (Tomo I). Bunos Aires: Editorial Biblos.

Devés Valdés, E. (2003). El pensamiento latinoamericano en el siglo XX: Desde la CEPAL al neoliberalismo, (1950-1990) (Tomo II). Buenos Aires: Editorial Biblos.

Devés Valdés, E. (2004). El pensamiento latinoamericano en el siglo XX: Entre la modernización y la identidad (Tomo III). Buenos Aires: Editorial Biblos.

Devés Valdés, E. (2007). Pensar (en) Paraguay: Los desafios de la intelectualidad paraguaya. En Beatriz González de Bosio, y Eduardo Devés-Valdés (coordinadores), Pensamiento Paraguayo del siglo XX (pp. 299-332). Asunción: Intercontinental.

Galeano, L. A. (2002). La sociedad dislocada. Asunción: CPES.

Garretón, M. A., Murmis, M., De Sierra, G., y González, H., (Compiladores). (2000). Historia crítica de la sociología argentina: Los raros, los clásicos, los científicos, los discrepantes. Buenos Aires: Colihue Universidad Sociología.

Heisecke, G. (1965). La Bibliografia sociológica en el Paraguay. Revista Paraguaya de Sociología, Año I(2).

Meliá, B., y Palau, T. (1975). Producción sociológica sobre el Paraguay: Relevamiento bibliográfico de los últimos años. Estudios Paraguayos, III(1), 147-173.

Murga Frassinetti, A., y Boils, G. (1979). Las ciencias sociales en América Latina. D.F.México: Universidad Nacional Autónoma de México, Facultad de Ciencias Políticas y Sociales.

Neiburg, F. y Plotkin, M. (editores). (2004). Intelectuales y expertos: La construcción del conocimiento social en la Argentina. Buenos Aires: Paidós.

Noé, M., y Dávila, A. (2005). Utopía y desencanto: Creación e institucionalización de la Carrera de Sociología en la Universidad de Buenos Aires 1955-1966. Buenos Aires.

Pereyra, D. (2007). Cincuenta años de la carrera de sociología de la UBA: Algunas notas contra-celebratorias para repensar la historia de la sociología en la Argentina. Revista Argentina de Sociología, 5(009), 153-159. Recuperado de http://redalyc.uaemex.mx

Pereyra, D. (2008). Sociografía, sociología e investigación social en Tucumán durante el peronismo: El Instituto de Sociografía y Planeación (1940-1957). Ponencia presentada en el Primer Congreso de estudios sobre el peronismo: La primera década Mar del Plata, Noviembre. Eje: Sociedad y cultura. (Coordinadores: Omar Acha- Gustavo Rubinstein).

Pereyra, D. (Compilador). (2010). El desarrollo de las ciencias sociales: Tradiciones, actores e instituciones en Argentina, Chile, México y Centroamérica. Cuaderno de Ciencias Sociales, 153.

Reyna, J. L. (2004). La institucionalización y profesionalización de las ciencias sociales en Américo Latina. Estudios Sociológicos, XXII(002), 483-493. Recuperado de http://redalyc.uaemex.mx

Schuttenberg, M. (2006). Sociología "científica” y ensayismo nacional popular: Un análisis de la discusión a partir de las obras de Gino Germani, Jauretche y Hernández Arregui. Utopias, 19-43.

Trindade, H. (coord)., De Sierra, G. Garretón, M. A. Murmis, M., y Reyna, J. L. (2007) Las ciencias sociales en América Latina en perspectiva comparada. México: Siglo XXI. 